Maternal $H L A-G^{*} 01: 01: 01: 04$ protects from anti-HLA-class II immunization in pregnant 2 women

3

4

Mohamed Slimane ${ }^{1}$, Christophe Picard ${ }^{2,3}$, Jacques Chiaroni ${ }^{2,3}$, Anderson Loundou ${ }^{4}$, Julien Paganini $^{5}$, Pierre Tiberghien ${ }^{1,6}$, Jean-Michel Rebibou ${ }^{1,7}$, Julie Di Cristofaro ${ }^{2,3}$

1. UMR 1098, Inserm, Université de Franche-Comté, Etablissement Français du Sang BFC, IFR 133, Besançon, France

2. Aix Marseille Univ, CNRS, EFS, ADES, "Biologie des Groupes Sanguins", Marseille, France

3. Etablissement Français du Sang PACA Corse, Marseille, France

4. Department of Public Health -EA 3279 Research Unit, University Hospital Marseille, AixMarseille University, Marseille, France

5. Xegen, Gemenos, France

6. Laboratoire d'Immunogénétique, Établissement Français du Sang BFC, Besançon, France

7. Service de Néphrologie, Centre Hospitalier Universitaire, Dijon, France

Corresponding author: julie.dicristofaro@efs.sante.fr

Abbreviated Title: $H L A-G$ haplotypes and anti-HLA immunization

Keywords: HLA-G, haplotypes, anti-HLA imunization, regulatory regions, pregnancy

Abbreviations: HLA: Human Leukocyte Antigen; CTL: Cytotoxic T-Lymphocyte; NK: Natural Killer cells; ILT: Immunoglobulin-Like Transcript; KIR: Killer-cell Immunoglobulinlike Receptor; sHLA-G: soluble HLA-G; UTR: UnTranslated Region; URR: Upstream Regulatory Region; gDNA: genomic DNA 


\begin{abstract}
2 Factors determining anti-HLA immunization are poorly understood, although anti-HLA

3 immunization following pregnancy is well described.

4 The HLA-G molecule has been extensively described for its implication in immunological

5 tolerance, especially during pregnancy. Transplant studies show an association between $H L A$ -

$6 \quad G$ haplotypes and alloimmunization.

7 Our aim was to investigate the association of $H L A-G$ haplotypes with anti-HLA class I and II

8 immunization in a cohort of women having experienced one or more pregnancies and with no 9 transfusion history.

10 Maternal blood samples $(\mathrm{n}=270)$ collected at delivery and formerly screened for anti-HLA 11 antibodies, HLA-A and HLA-B antigens, were screened by NGS for HLA-G gene 12 polymorphism.

13 Univariate analysis further confirmed that the number of pregnancies was significantly 14 associated with anti-HLA class I immunization, whereas no other variable remained 15 significant after Bonferroni correction. Our results showed however that anti-HLA class II 16 immunization was associated with the number of children whereas the HLA-G*01:01:01:04 17 allele was protective against this immunization.
\end{abstract}


The HLA-G molecule has been extensively described for its implication in immunological tolerance, especially during pregnancy. Although it displays a restricted number of alleles, many studies associated $H L A-G$ alleles with differential level of tolerance in achallenging immune situations such as pregnancies and transplantation outcome. Indeed $H L A-G$ displays few haplotypes (including 5'URR, coding and 3'UTR regions) associated with differential protein expression patterns [1] and differential clinical outcome of immunosensitive events. $H L A-G$ alleles were associated with pregnancy complications [1] and recurrent miscarriages [1] as well as with immunization and poor prognosis in lung transplant patients [1].

10

Alloimmunization, especially against HLA molecules, is also responsible for adverse effects and acute rejection in blood transfusion and organ transplantation; including febrile nonhaemolytic transfusion reactions, immunological platelet refractoriness or transfusion-related acute lung injury. The main cause of naturally occurring anti-HLA immunization is pregnancy, with an extensively confirmed increased HLA antibody prevalence with a greater number of pregnancies [1]. Anti-HLA antibodies are the most studied antibodies, but little is known about non-HLA antibodies mainly directed against endothelial cells, and although this field has been intensively studied, their prevalence and implication in physio-pathological process remain unclear [1].

Biological and genetic factors determining anti-HLA immunization remain poorly understood with the exception of HLA-A, B or DR antigens or eplets [1]. HLA immunogenicity during pregnancy was found to be lower in women who experienced a prior miscarriage compared to women who had a prior successful pregnancy [2]. Masson et al. analyzed genetic polymorphisms implicated in humoral immunization, and none of the following parameters influenced immunization rate: HLA-typing, TLR4 gene polymorphisms D299G, T399I; IL-6 promoter polymorphism $-174 \mathrm{C} / \mathrm{G}$ and $B A F F$ intron $\mathrm{G} / \mathrm{T}$ and promoter polymorphism -871 $\mathrm{C} / \mathrm{T}$ [1]. In women having had only one successful pregnancy, there was a significant association between $I L-6$ promoter $-174 \mathrm{G} / \mathrm{C}$ and immunization [1]. HLA-G expression was found to be lower in women with antibodies detectable both by CDC and Luminex than in women with antibodies detected only by Luminex [1]. Such serological testing is however poorly reproducible [3], whereas most HLA laboratories nowadays have DNA analysis facilities making $H L A-G$ studies a feasible option. Thus, $H L A-G$ molecule may represent a good complementary genetic predictive factor for anti-HLA immunization.

To get further insight into the association of $H L A-G$ haplotypes with immunization, we recently investigated $H L A-G$ phylogeny with regards to inflammatory response [1]. Our results split $H L A-G$ haplotypes phylogenetically into four main clades respectively containing (1) $H L A-G^{*} 01: 01: 02: 01, G^{*} 01: 01: 02: 02, G^{*} 01: 05 N$ and $G^{*} 01: 06$ (associated with haplotype

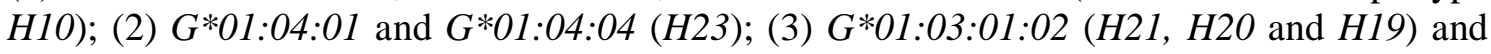

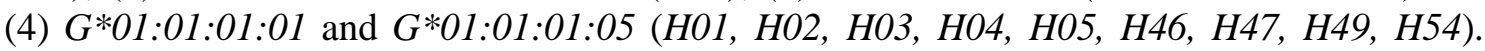
Whereas $H L A-G$ alleles associated with poor prognosis in transplantation, pregnancy or inflammatory disease were grouped in clades 1,2 and 3, some of the $H L A-G$ alleles in the fourth clade were associated with a protective effect against inflammation [1].

The main goal of this study was to confirm the association of $H L A-G$ phylogeny with differential immunological tolerance induction. We thus investigated $H L A-G$ haplotype association with the presence of anti-HLA antibodies in women who had experienced one or more pregnancies but who had no transfusion history. 


\section{Population and sample collection}

3 Two hundred and seventy women having had one or more pregnancies were included in the

4 study. None of these women had any history of transfusion. Maternal blood samples and 5 umbilical cord samples were collected at delivery [1]. Patient data (age, number of children, 6 pregnancies and miscarriages) are given in Table 1. The study was designed in collaboration with the Besançon Cord Blood Bank (Etablissement Français du Sang) and carried out in accordance with the recommendations of Besançon Hospital's Ethics Committee with written informed consent from all subjects. All subjects gave written informed consent in accordance with the Declaration of Helsinki. The protocol was approved by the Besançon Hospital's Ethics Committee.

\section{$H L A-G$ analysis and HLA typing}

Maternal DNA was sequenced for $H L A-G$ gene from position -1983 to +3447 by Next Generation Sequencing (NGS). PCR fragments were sequenced using an NGS platform (MiSeq, Illumina, The Netherlands) [1].

HLA-G NGS data were analyzed using PolyPheMe (Xegen, France). HLA-G allelic assignment at 8 digits was based on the HLA sequences listed in the official IMGT/HLA database 3.28.0 [4]. All polymorphic variations from position -1983 to -1 in 5'URR and from +2540 to +3447 in 3'UTR, i.e. 37 SNPs, 1 base deletion, 1 base insertion and a 14bp insertion were used for haplotype estimation using PHASE. $H L A-G$ haplotype identification numbers were coded according to [1]. Allelic and haplotype frequencies were estimated using an EM algorithm implemented in the Gene [Rate] computer tools [5].

Both maternal and child DNA were typed for HLA-A and HLA-B at a low resolution level by Luminex $^{\mathrm{TM}}$ technology (One Lambda LABType ${ }^{\circledR}$ SSO, InGen, France), complete procedure is described in [1]. HLA-C, DR or DQ were not investigated.

Latest child/paternal HLA-A and HLA-B mismatch were deduced from mother and latest child HLA-A and HLA-B typing results. Maternal IL-6 -174 C/G genotype results obtained by Snap Shot were included in this study, complete procedure is described in [1].

\section{HLA antibody testing}

Each serum was screened for anti-HLA antibodies using two techniques: the standard CDC method (for anti-class I antibodies) and the Luminex screening kits (for anti-class I and class II antibodies), complete procedure is described in [1].

\section{Statistical analysis}

Missing data led to the exclusion of the concerned sample from further analyses. No multiple imputation was used. Analysis were performed using GRAPH PAD Prism 5 software (CA, www.graphpad.com), SPSS 24.0 software (SPSS Inc., Chicago) and SAS 9.4 (SAS Institute Inc. Cary, USA).

Biological and genetic data frequencies between anti-HLA class I immunized women, antiHLA class II immunized women and non-immunized women were compared with Chi-square tests. The presence of anti-HLA class I antibodies and anti-HLA class II antibodies were independently tested in univariate analyses according to biological data (women's age, 
1 number of pregnancies, number of children delivered, number of miscarriages, sex of the 2 latest child) and according to genetic data (HLA-G alleles and $H L A-G$ haplotypes, $I L-6-174$

3 C/G genotype, HLA-A and HLA-B antigen, latest child/paternal HLA-A and HLA-B antigen 4 mismatch). HLA-A and HLA-B antigens and latest child/paternal HLA-A and HLA-B 5 mismatches were included for their association with allo-immunization because of the 6 Linkage Disequilibrium between HLA-G and HLA-A loci and HLA-A and HLA-B [1]. IL-6 $7 \quad 174 \mathrm{C} / \mathrm{G}$ genotype was included in the study since it was described as being significantly 8 associated with immunization in women having had only one successful pregnancy [1].

9 Bonferroni correction for multiplicity testing procedure was applied and variables with p10 values below 0.001 in univariate analyses were further investigated in multivariate regression analysis. The hazard ratios were estimated by using Firth's penalized partial likelihood approach. Association results are expressed as odds ratio (OR) and confidence intervals of 95\% (CI 95\%).

\section{RESULTS}

Anti-HLA class I and class II immunization data according to biological characteristics are shown in Table 1. Univariate analyses showed no difference between anti-HLA-I immunized and anti-HLA-I non-immunized women, or between anti-HLA-II immunized and anti-HLA-II non-immunized women according to age or gender of latest child. Anti-HLA-I and anti-HLAII immunization were associated both with the number of pregnancies and the number of children (both $\mathrm{p}<0.0001$ ) but not with miscarriages; the association of miscarriages with antiHLA-II immunization did not reach significance after Bonferroni correction $(\mathrm{p}=0.007)$.

$H L A-G$ allelic frequency and characteristics of estimated $H L A-G$ haplotypes with a frequency above $1 \%$ are shown in Table 2 . Most $H L A-G$ haplotypes with a frequency above $1 \%$ display an exclusive association with an $H L A-G$ allele defined at high resolution, i.e. 8 digits.

The characteristics of anti-HLA class I and class II antibody groups according to HLA-G alleles and haplotypes are shown in Table 3.

Univariate analyses showed no significant difference between anti-HLA-I immunized and anti-HLA-I non-immunized women according to HLA-A and HLA-B antigens (data not shown), IL-6 G/C genotype (data not shown), HLA-G allele or haplotype, and latest child/paternal HLA-A and HLA-B mismatch (data not shown) after Bonferroni correction.

None of these variables displayed any significant differences either between women with antiHLA-II antibodies and anti-HLA-II non-immunized women (data not shown), except the HLA-G allele $G^{*}$ 01:01:01:04 and haplotype H03. This allele was never observed in antiHLA-II immunized women whereas it were present in $11.5 \%$ of anti-HLA-II non-immunized women.

Of note, variables that did not reach significance after Bonferroni correction and were thus not included in multivariate analyses were the following for anti-HLA class I antibody presence: H03-G*01:01:01:04, $\mathrm{B}^{*} 13$ and $\mathrm{B} 38$, and for anti-HLA class II presence: H19$G^{*} 01: 03: 01: 02, \mathrm{~A} * 29, \mathrm{~A} * 31, \mathrm{~B} * 18, \mathrm{~B} * 57, \mathrm{~B} * 60$, paternal mismatch $\mathrm{B} * 51$ and paternal mismatch $\mathrm{B}^{*} 62$ (Table 2). It should be noted that $H L A-G^{*} 01: 03: 01: 02$ was the only allele observed for $H L A-G^{*} 01: 03$.

Multivariate analysis was performed by logistic regression hazard modeling using Firth's penalized partial likelihood approach with variables showing significant p-values after 
1 Bonferroni correction. One variable of those displaying high correlation was chosen (either 2 number of pregnancies or number of children for biological variables, and either $\mathrm{HO}$ or $3 G^{*}$ 01:01:01:04 for genetic variables). Anti-HLA class II immunization displayed significant 4 association with number of children ( $\mathrm{p}<0.0001$, hazard ratio: 1.725; CI 95\%: 1.1339-2.223) 5 whereas $G^{*}$ 01:01:01:04 allele was significantly protective against anti-HLA class II 6 immunization ( $\mathrm{p}=0.0422$, hazard ratio: 0.0422; CI 95\%: 0.003-0.0899) (Table 4).

\section{DISCUSSION}

Anti-HLA immunization is a main issue in blood transfusion and organ transplantation. Pregnancy is the main source of HLA sensitizing events: half to two thirds of pregnant women seem to develop anti-HLA antibodies [1].

HLA-G is extensively reported to play a pivotal role in fetomaternal tolerance and transplantation outcome [3]. We previously showed that $H L A-G$ haplotypes, i.e. SNPs associated in Linkage Disequilibrium, (1) remain preserved between populations with different geographical origins, (2) can reliably be used to predict sHLA-G levels, (3) are associated with alloimmunization and inflammation in transplantation and inflammatory disease and (4) are grouped into four main clades, three of which contain alleles associated with lower immunotolerance whereas the fourth seems to be associated with higher immunotolerance [1].

In this study, we aimed to confirm whether $H L A-G$ haplotypes could be a predictive genetic risk or protective factor for anti-HLA immunization and may help clinicians to estimate the risk of rejection in each single case, while considering the consequences of performing the graft or not.

We thus investigated $H L A-G$ haplotype association with anti-HLA immunization in women having experienced pregnancy but with no transfusion or transplant history.

We performed $H L A-G$ genetic analysis on 270 maternal blood samples collected at delivery and tested for anti-HLA antibodies. Statistical analysis included variables reported to be associated with anti-HLA immunization such as the number of pregnancies, number of children, number of miscarriages, HLA-A and HLA-B antigen typing and paternal HLA-A and HLA-B mismatch.

We found that H03-HLA-G*01:01:01:04 was the only genetic variable to remain significantly associated with anti-HLA class II immunization after Bonferroni correction ( $p>0.001)$. Its protective value against anti-HLA class II immunization remained significant in multivariate analysis including the number of pregnancies $(\mathrm{p}=0.0422)$. However, it should be noted that since only 22 women presented $H L A-G^{*} 01: 01: 01: 04$, an accidental better HLA class II matching of mothers and children in this group is not excluded. Furthermore, when anti-HLA class I immunization is considered, for which latest child/paternal mismatch displayed no significant results, this same HLA-G allele was found to be protective $(p=0.031)$ but it was not included in multivariate analysis because of Bonferroni correction.

Although this study was not designed to decipher the biological mechanism leading to our results, it seems to support our previous work on phylogenetic analysis of $H L A-G$ alleles defined at high resolution. Phylogenetic structure of $H L A-G$ sequences consisted of four main clades which may reflect HLA-G tolerogenic properties; indeed each clade displayed specific transcription factor sites and coding sequence variations [1]. The conservation of HLA-G sequences worldwide suggests that those which lowered immunotolerance might provide an 
1 advantage in specific contexts [6]. Tolerogenicity would come from HLA-G binding with the 2 ILT-2 receptor on $\mathrm{B}$ cells and thus limiting their proliferation, differentiation and 3 immunoglobulin secretion [1].

4 In pregnancy, reduced expression of HLA-G seemed to be associated with complications, 5 such as miscarriage or preeclampsia. Interestingly, women with preeclampsia harbor higher 6 concentrations of cellular fetal microchimerism and anti-paternal allo-antibodies [1].

7 Masson et al. showed, in the same cohort as this study, that anti-HLA immunization increased 8 with the number of pregnancies and of children delivered but they could not find any 9 association with HLA-A and HLA-B antigens. Only the $-174 \mathrm{G} / \mathrm{C}$ polymorphism in the IL-6 10 promoter (located on chromosome 7) was associated with a lower risk of anti-HLA antibody 11 development among primiparous pregnant women with no history of miscarriage [1]. Our analysis further confirmed their main results. We did not find any association with HLA-A, HLA-B or their paternal mismatch, on the contrary to Piacasdia et al. who showed that HLA$\mathrm{B} * 14$ and HLA-B*51 were associated with a lower risk of anti-HLA antibody development after pregnancy, while having the $A * 11$ allele seemed to represent a higher risk in a cohort of 161 pregnancy-only sensitizing events [1].

17 This preliminary study seems to confirm the role of HLA-G in inflammation and immune control; it needs however to be completed by a larger number of patients to get a better insight into the nature of the association with each individual HLA locus. We previously reported a strong linkage disequilibrium of both $H L A-A$ and $H L A-F$ with $H L A-G$ [1]; such extended HLA class I haplotypes may represent even more pertinent predictive values, notably HLA-F which is shown to play an important part in immune regulation [7]. This descriptive study would also benefit from investigating into biological mechanisms leading the association of $H L A-G$ phylogeny with differential immunological tolerance induction. 


\section{CONFLICT OF INTEREST}

2 The authors declare that they have no conflict of interest.

\section{AUTHOR CONTRIBUTIONS}

4 Conception or design of the work: MS, CP, JC, PT, JMR, JDC

5 Acquisition, analysis and interpretation of data: MS, CP, JC, AD, JP, PT, JMR, JDC

6 Drafting the work or revising it critically for important intellectual content: MS, CP, JC, AD,

7 JP, PT, JMR, JDC

8 Final approval of the version to be published MS, CP, JC, AD, JP, PT, JMR, JDC

9 Agreement to be accountable for all aspects of the work in ensuring that questions related to

10 the accuracy or integrity of any part of the work are appropriately investigated and resolved:

11 MS, CP, JC, AD, JP, PT, JMR, JDC

12 FUNDING

13 This study was supported by Etablissement Français du Sang.

\section{ACKNOWLEDGEMENT}

15 The authors would like to thank the donors. 
Table 1. Women's data under study. Age, sex of latest child, number of pregnancies, children and miscarriages are given for all women, anti-HLA class I immunized, anti-HLA class I non-immunized, anti-HLA class II immunized and anti-HLA class II non-immunized women; p-values under 0.001 are in bold.

\begin{tabular}{|c|c|c|c|c|c|c|c|}
\hline & All & $\begin{array}{l}\text { Anti-HLA class } \\
\text { I immunized }\end{array}$ & $\begin{array}{l}\text { Anti-HLA class I } \\
\text { non-immunized }\end{array}$ & p-value & $\begin{array}{l}\text { Anti-HLA class II } \\
\text { immunized }\end{array}$ & $\begin{array}{c}\text { Anti-HLA class II } \\
\text { non-immunized }\end{array}$ & $\mathrm{p}$-value \\
\hline Number of patients (\%) & 270 & $100(37.1)$ & $170(62.9)$ & & $70(26.0)$ & $200(74.0)$ & \\
\hline Age (yrs) (median and range) & $29.3[18-42]$ & 29.7 [19-40] & $29.0[18-42]$ & 0.176 & 29.9 [19-41] & $29.0[18-42]$ & 0.209 \\
\hline Sex of latest child sex (Male \%) & 51.5 & 55 & 49.4 & 0.375 & 59.4 & 48.8 & 0.126 \\
\hline Pregnancies (median and range) & $2.1[1-6]$ & $2.4[1-6]$ & $1.9[1-6]$ & $>0.001$ & $2.6[1-6]$ & $1.9[1-6]$ & $>0.001$ \\
\hline $1(\%)$ & 35.2 & 20.0 & 44.1 & $>0.001$ & 14.5 & 42.3 & $>0.001$ \\
\hline $2(\%)$ & 36.3 & 45.0 & 31.2 & 0.022 & 39.1 & 35.3 & 0.570 \\
\hline $3(\%)$ & 15.2 & 16.0 & 14.7 & 0.774 & 23.2 & 12.4 & 0.032 \\
\hline $4(\%)$ & 10.0 & 14.0 & 7.6 & 0.092 & 17.4 & 7.5 & 0.018 \\
\hline $5(\%)$ & 2.6 & 4.0 & 1.8 & 0.264 & 4.3 & 2.0 & 0.288 \\
\hline $6(\%)$ & 0.7 & 1.0 & 0.6 & 0.703 & 1.4 & 0.5 & 0.426 \\
\hline Children (median and range) & $1.6[1-5]$ & $1.96[1-4]$ & $1.5[1-5]$ & $>0.001$ & $2.0[1-4]$ & $1.58[1-5]$ & $>0.001$ \\
\hline $1(\%)$ & 48.1 & 31.0 & 58.2 & $>0.001$ & 26.1 & 55.7 & $>0.001$ \\
\hline $2(\%)$ & 38.1 & 49.0 & 31.8 & 0.005 & 50.7 & 33.8 & 0.013 \\
\hline $3(\%)$ & 10.0 & 13.0 & 8.2 & 0.208 & 17.4 & 7.5 & 0.018 \\
\hline $4(\%)$ & 3.3 & 7.0 & 1.2 & 0.010 & 5.8 & 2.5 & 0.186 \\
\hline $5(\%)$ & 0.4 & 0.0 & 0.6 & 0.442 & 0.0 & 0.5 & 0.557 \\
\hline Miscarriages (median and range) & $0.41[0-3]$ & $0.44[0-2]$ & $0.39[0-3]$ & 0.437 & $0.59[0-2]$ & $0.35[0-5]$ & 0.007 \\
\hline $0(\%)$ & 67.0 & 64.0 & 68.8 & 0.416 & 55.1 & 71.1 & 0.014 \\
\hline
\end{tabular}




\begin{tabular}{|l|c|c|c|c|c|c|c|}
\hline $1(\%)$ & 25.6 & 28.0 & 24.1 & 0.480 & 30.4 & 23.9 \\
\hline $2(\%)$ & 6.7 & 8.0 & 5.9 & 0.501 & 14.5 & 4.0 \\
\hline $3(\%)$ & 0.7 & 0.0 & 1.2 & 0.276 & 0.0 & 0.003 \\
\hline
\end{tabular}


1 Table 2. Characteristics of $H L A-G$ haplotypes estimated with a frequency above 1\% (ID: identification number according to [1], N: Number, Fq:

2 Frequency).

3

\begin{tabular}{|c|c|c|c|c|c|}
\hline $\begin{array}{l}\text { Haplotype } \\
\text { ID }\end{array}$ & 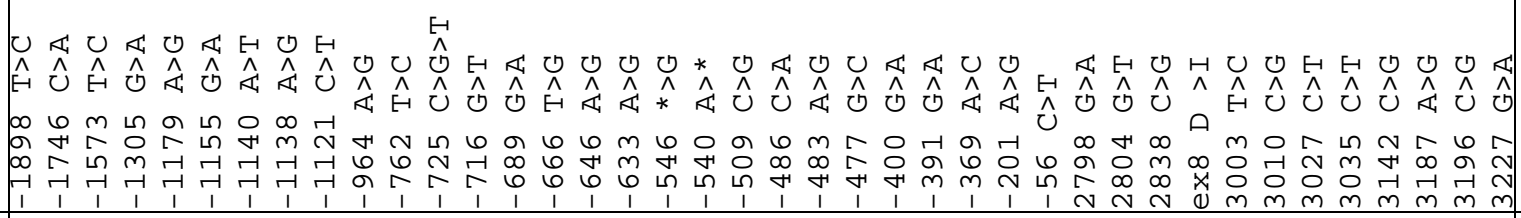 & $\begin{array}{c}\text { Associated } \\
\text { allele }\end{array}$ & $\begin{array}{c}\text { GenBank } \\
\text { accession } \\
\text { number }\end{array}$ & $N$ & $\boldsymbol{F q}$ \\
\hline HO1 & 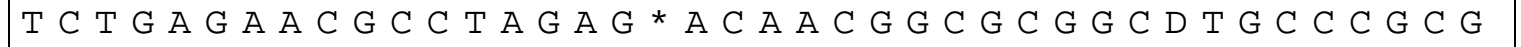 & $G^{*} 01: 01: 01: 01$ & MG825364 & 145 & 26.9 \\
\hline \multirow{4}{*}{$H 10$} & \multirow{4}{*}{ 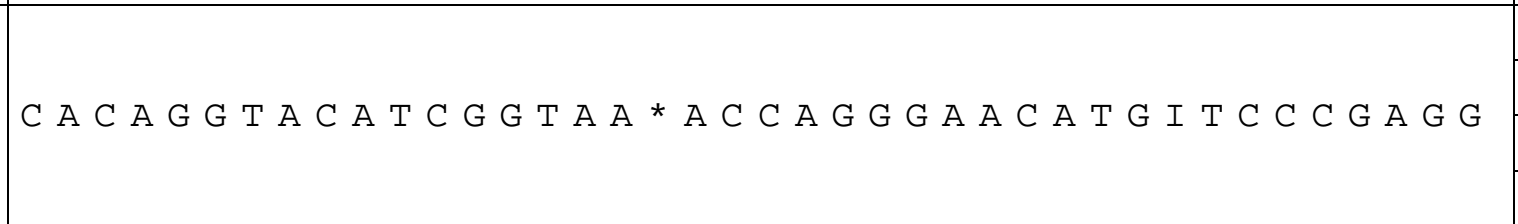 } & G*01:01:02:01 & MG825359 & 112 & 20.7 \\
\hline & & $G^{*} 01: 06$ & MG825360 & 28 & 5.2 \\
\hline & & $G^{*} 01: 05 N$ & MG825361 & 12 & 2.2 \\
\hline & & $G^{* 01: 01: 02: 02}$ & MG825362 & 3 & 0.6 \\
\hline HO4 & 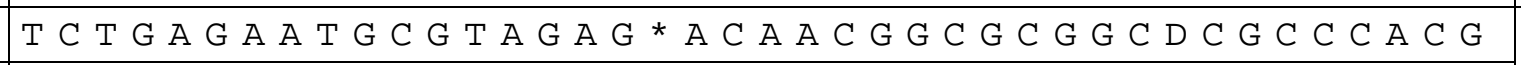 & \multirow{2}{*}{$G^{*} 01: 01: 01: 05$} & \multirow{2}{*}{$\begin{array}{l}\text { MG825356 } \\
\text { MG825358 }\end{array}$} & 46 & 8.5 \\
\hline HO2 & 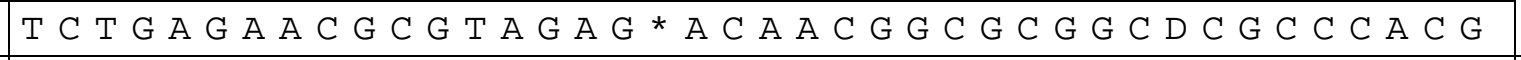 & & & 38 & 7.0 \\
\hline \multirow{2}{*}{$H 23$} & \multirow{2}{*}{ 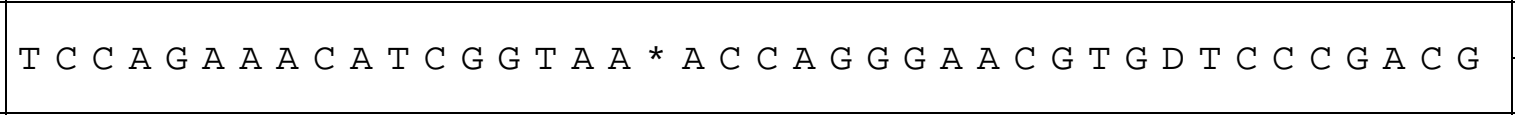 } & $G^{*} 01: 04: 01$ & MG825349 & 37 & 6.9 \\
\hline & & $G^{*} 01: 04: 04$ & MG825348 & 7 & 1.3 \\
\hline$H 16$ & 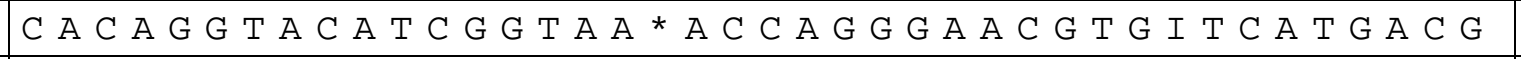 & $G^{* 01: 01: 03: 03}$ & MG825347 & 22 & 4.1 \\
\hline HO3 & T C T G A GA A C G C C TAGA $A \star \star C A A C G G C G C G G C D T G C C C A C A$ & $G^{* 01: 01: 01: 04}$ & MG825363 & 22 & 4.1 \\
\hline$H 21$ & 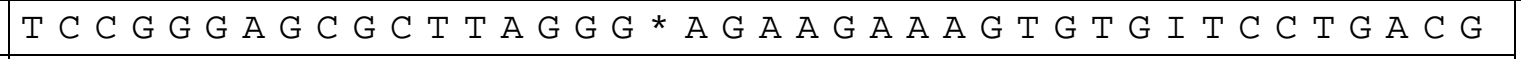 & \multirow{3}{*}{$G^{* 01: 03: 01: 02}$} & \multirow{3}{*}{$\begin{array}{l}\text { MG825350 } \\
\text { MG825354 } \\
\text { MG825357 }\end{array}$} & 8 & 1.5 \\
\hline$H 20$ & 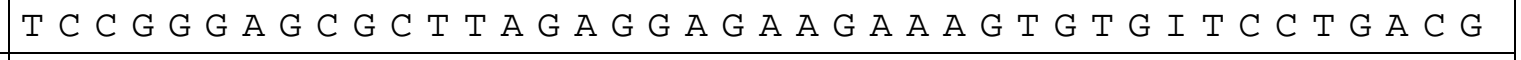 & & & 8 & 1.5 \\
\hline$H 19$ & T C C G G A G C G C T TA A A GACA A GA A A T G T I T C C T G A C G & & & 7 & 1.3 \\
\hline
\end{tabular}


Table 3. Characteristics of immunized and non-immunized anti-HLA class I and anti-HLA class II groups for $H L A-G$ allele and haplotype frequencies; p-values under 0.001 are in bold.

\begin{tabular}{|c|c|c|c|c|c|c|c|}
\hline & All & $\begin{array}{l}\text { Anti-HLA class I } \\
\text { non-immunized }\end{array}$ & $\begin{array}{l}\text { Anti-HLA class I } \\
\text { immunized }\end{array}$ & p-value & $\begin{array}{l}\text { Anti-HLA class II } \\
\text { non-immunized }\end{array}$ & $\begin{array}{l}\text { Anti-HLA class II } \\
\text { immunized }\end{array}$ & p-value \\
\hline \multicolumn{8}{|c|}{$H L A-G$ allele carrier } \\
\hline$G * 01: 01: 01: 01$ & 49.3 & 51.8 & 45.0 & 0.172 & 52.0 & 41.4 & 0.105 \\
\hline$G * 01: 01: 01: 02$ & 0.4 & 0.0 & 1.0 & 0.370 & 0.0 & 1.4 & 0.256 \\
\hline$G * 01: 01: 01: 03$ & 0.7 & 1.2 & 0.0 & 0.396 & 1.0 & 0.0 & 0.553 \\
\hline$G * 01: 01: 01: 04$ & 8.5 & 11.2 & 4.0 & 0.031 & 11.5 & 0.0 & 0.001 \\
\hline$G * 01: 01: 01: 05$ & 28.5 & 26.5 & 32.0 & 0.202 & 28.0 & 30.0 & 0.396 \\
\hline$G * 01: 01: 01: 06$ & 0.4 & 0.0 & 1.0 & 0.370 & 0.0 & 1.4 & 0.256 \\
\hline$G * 01: 01: 02: 01$ & 38.1 & 38.2 & 38.0 & 0.537 & 38.0 & 38.6 & 0.477 \\
\hline$G * 01: 01: 02: 02$ & 1.1 & 1.8 & 0.0 & 0.248 & 1.5 & 0.0 & 0.411 \\
\hline$G * 01: 01: 03: 03$ & 8.5 & 8.8 & 8.0 & 0.503 & 9.5 & 5.7 & 0.252 \\
\hline$G * 01: 01: 05$ & 0.4 & 0.6 & 0.0 & 0.630 & 0.5 & 0.0 & 0.744 \\
\hline$G * 01: 01: 12$ & 0.4 & 0.6 & 0.0 & 0.630 & 0.5 & 0.0 & 0.744 \\
\hline$G * 01: 01: 15$ & 0.4 & 0.6 & 0.0 & 0.630 & 0.5 & 0.0 & 0.744 \\
\hline$G * 01: 03: 01: 02$ & 8.1 & 8.2 & 8.0 & 0.571 & 6.5 & 12.9 & 0.075 \\
\hline$G * 01: 04: 01$ & 16.3 & 15.3 & 18.0 & 0.338 & 16.0 & 17.1 & 0.453 \\
\hline$G * 01: 04: 04$ & 3.0 & 2.4 & 4.0 & 0.337 & 2.0 & 5.7 & 0.118 \\
\hline$G * 01: 05 N$ & 4.4 & 5.9 & 2.0 & 0.114 & 5.5 & 1.4 & 0.142 \\
\hline$G * 01: 06$ & 10.7 & 8.8 & 14.0 & 0.131 & 11.0 & 10.0 & 0.528 \\
\hline$G * 01: 10$ & 0.7 & 1.2 & 0.0 & 0.396 & 1.0 & 0.0 & 0.553 \\
\hline \multicolumn{8}{|c|}{$H L A-G$ haplotype carrier } \\
\hline H01 & 46.7 & 49.4 & 42.0 & 0.146 & 48.0 & 42.9 & 0.318 \\
\hline HO2 & 14.1 & 12.4 & 17.0 & 0.189 & 13.0 & 17.1 & 0.233 \\
\hline
\end{tabular}




\begin{tabular}{|c|c|c|c|c|c|c|c|}
\hline H03 & 8.1 & 10.6 & 4.0 & 0.042 & 11.0 & 0.0 & 0.001 \\
\hline HO4 & 16.7 & 15.9 & 18.0 & 0.386 & 16.5 & 17.1 & 0.492 \\
\hline H05 & 1.9 & 1.8 & 2.0 & 0.612 & 2.0 & 1.4 & 0.621 \\
\hline H06 & 0.4 & 0.0 & 1.0 & 0.370 & 0.0 & 1.4 & 0.256 \\
\hline H08 & 0.4 & 0.6 & 0.0 & 0.630 & 0.5 & 0.0 & 0.744 \\
\hline H09 & 0.4 & 0.6 & 0.0 & 0.630 & 0.5 & 0.0 & 0.744 \\
\hline H10 & 50.0 & 48.8 & 52.0 & 0.353 & 50.5 & 48.6 & 0.500 \\
\hline H11 & 0.4 & 0.0 & 1.0 & 0.370 & 0.0 & 1.4 & 0.256 \\
\hline H13 & 1.1 & 1.8 & 0.0 & 0.248 & 1.5 & 0.0 & 0.411 \\
\hline H16 & 8.1 & 8.2 & 8.0 & 0.571 & 9.0 & 5.7 & 0.293 \\
\hline H19 & 2.2 & 1.2 & 4.0 & 0.138 & 0.5 & 7.1 & 0.005 \\
\hline $\mathrm{H} 20$ & 3.3 & 4.7 & 1.0 & 0.094 & 3.5 & 2.9 & 0.585 \\
\hline H21 & 3.0 & 2.9 & 3.0 & 0.622 & 2.5 & 4.3 & 0.334 \\
\hline $\mathrm{H} 23$ & 16.3 & 15.3 & 18.0 & 0.338 & 15.5 & 18.6 & 0.312 \\
\hline H26 & 0.4 & 0.0 & 1.0 & 0.370 & 0.5 & 0.0 & 0.744 \\
\hline $\mathrm{H} 28$ & 0.4 & 0.6 & 0.0 & 0.630 & 0.5 & 0.0 & 0.744 \\
\hline H31 & 0.4 & 0.6 & 0.0 & 0.630 & 0.5 & 0.0 & 0.744 \\
\hline H32 & 0.4 & 0.6 & 0.0 & 0.630 & 0.5 & 0.0 & 0.744 \\
\hline H36 & 0.7 & 0.6 & 1.0 & 0.604 & 0.5 & 1.4 & 0.447 \\
\hline H38 & 0.4 & 0.6 & 0.0 & 0.630 & 0.5 & 0.0 & 0.744 \\
\hline H44 & 0.4 & 0.6 & 0.0 & 0.630 & 0.5 & 0.0 & 0.744 \\
\hline H46 & 1.1 & 0.6 & 2.0 & 0.309 & 1.5 & 0.0 & 0.411 \\
\hline $\mathrm{H} 48$ & 0.4 & 0.6 & 0.0 & 0.630 & 0.5 & 0.0 & 0.744 \\
\hline H49 & 0.4 & 0.0 & 1.0 & 0.370 & 0.0 & 1.4 & 0.256 \\
\hline H52 & 0.4 & 0.6 & 0.0 & 0.630 & 0.5 & 0.0 & 0.744 \\
\hline H53 & 0.4 & 0.6 & 0.0 & 0.630 & 0.5 & 0.0 & 0.744 \\
\hline H54 & 0.7 & 0.0 & 2.0 & 0.136 & 1.0 & 0.0 & 0.553 \\
\hline
\end{tabular}




\begin{tabular}{|l|l|l|l|l|l|l|l|}
\hline H56 & 0.4 & 0.6 & 0.0 & 0.630 & 0.5 & 0.0 \\
\hline H58 & 0.4 & 0.0 & 1.0 & 0.370 & 0.0 & 0.744 \\
\hline H61 & 0.4 & 0.6 & 0.0 & 0.630 & 0.5 & 0.4 & 0.0 \\
\hline H63 & 0.4 & 0.6 & 0.0 & 0.630 & 0.5 & 0.0 \\
\hline $\boldsymbol{H 7 1}$ & 0.4 & 0.6 & 0.0 & 0.630 & 0.5 & 0.0 & 0.7444 \\
\hline $\boldsymbol{H 7 2}$ & 0.4 & 0.6 & 0.0 & 0.630 & 0.5 & 0.0 \\
\hline $\boldsymbol{H 7 3}$ & 0.4 & 0.0 & 1.0 & 0.370 & 0.0 & 0.744 \\
\hline
\end{tabular}


1 Table 4. Associated factors for anti-HLA class II immunization according to logistic 2 regression on number of pregnancies and HLA-G*01:01:01:04 (HR, hazard ratio; CI, 3 confidence interval).

4

\begin{tabular}{|l|l|l|}
\hline & Estimated HR $(95 \% \mathrm{CI})$ & $\mathrm{p}$-value \\
\hline Pregnancies & $1.725(1.339-2.223)$ & 0.0001 \\
HLA-G*01:01:01:04 & $0.049(0.003-0.899)$ & 0.0422 \\
\hline
\end{tabular}

5 


\section{References}

3

4

1. $\quad$ !!! INVALID CITATION !!!

2. $\quad$ Geneugelijk, K., et al., A Previous Miscarriage and a Previous Successful Pregnancy Have a Different Impact on HLA Antibody Formation during a Subsequent Successful Pregnancy. Front Immunol, 2016. 7: p. 571.

3. Rebmann, V., et al., HLA-G as a Tolerogenic Molecule in Transplantation and Pregnancy. J Immunol Res, 2014. 2014: p. 297073.

4. Robinson, J., et al., The IPD and IMGT/HLA database: allele variant databases, in Nucleic Acids Res. 2015, The Author(s) 2014. Published by Oxford University Press on behalf of Nucleic Acids Research.: England. p. D423-31.

5. Nunes, J.M., et al., Analysis of the HLA population data (AHPD) submitted to the 15th International Histocompatibility/Immunogenetics Workshop by using the Gene[rate] computer tools accommodating ambiguous data (AHPD project report). Tissue Antigens, 2010. 76(1): p. 18-30.

6. Castelli, E.C., et al., A comprehensive study of polymorphic sites along the HLA-G gene: implication for gene regulation and evolution. Mol Biol Evol, 2011. 28(11): p. 3069-86.

7. Persson, G., et al., HLA class Ib in pregnancy and pregnancy-related disorders. Immunogenetics, 2017. 